\title{
PHARMACOLOGICAL PROPERTIES OF CM
}

\section{Biochemical-pharmacological properties}

Contrast agents influence the human organism not only because of their physical properties such as osmolality, viscosity and hydrophilicity but also because of their special chemical structure. Contrary to former beliefs, effects are not induced by free iodine, but by the contrast medium molecule. Hemodynamic, neurological and pseudoallergic effects are partially attributable to the chemical structure of contrast media molecules.

Several biochemical reactions are more or less related to the general tolerance of XCM.

\section{Protein binding}

Contrast agents show variable binding to albumin in blood plasma after intravascular administration. Albumin binding is strongly enhanced by a free/ unsubstituted position five of the benzene ring, which is intentionally the case in intravenous cholegraphics. Those substances show an albumin binding of up to $90 \%$ [28].

All uroangiographic contrast agents in use today are substituted in position five of the benzene ring and possess additional hydroxyl groups $(-\mathrm{OH})$ and other hydrophilic groups (-CON), which make protein binding practically impossible. 
The following percentages of plasma protein binding were found in a large comparative study: $14 \%$ for the ionic dimeric ioxaglinic acid, $8.8 \%$ for the ionic diatrizoic acid, and $4.3 \%$ and $1.8 \%$ for the dimeric nonionic contrast agents iodixanol and iotrolan. The nonionic iopromide showed an extremely low binding of $<1 \%$ [29].

\section{Complement activation}

Many clinical observations and experimental studies showed XCM to be able to activate factors of the complement system, the kinin system, other mediators, and eventually the blood coagulation system as well as the fibrinolytic system.

A radioimmunoassay (RIA) study shows that different ionic and nonionic contrast agents may in vitro activate fraction $\mathrm{C} 3$ of the complement system and additionally release anaphylatoxin $\mathrm{C} 3 \mathrm{a}$ and $\mathrm{C} 5 \mathrm{a}$ [30].

The exact mechanism of complement activation is still debated. However, it appears to occur without involvement of Ig-complexes via the alternative pathway [31]. 


\section{Enzyme inhibition}

Plasma enzymes such as ß- glucoronidase, lysozyme, alcohol dehydrogenase and glucose-6-phosphate dehydrogenase are unspecifically inhibited by binding with CM, without this inhibition being clinically relevant.

Conversely, inhibition of plasma acetycholinesterase by $\mathrm{CM}$ may contribute to pseudoallergic reactions such as urticaria, gastrointestinal symptoms and vasodilatation.

A comparison of iopromide and iopamidol at concentrations of 0 to $40 \mathrm{mgl} / \mathrm{ml}$ showed the inhibition of acetylcholinesterase to be dose-dependent with $15 \%$ inhibition at the highest concentration of both CM [32].

Angiotensin-converting enzyme (ACE) is equally strongly inhibited by ionic and nonionic XCM in in-vitro studies. Inhibition is determined by the chelates used as stabilizers in the contrast agents.

ACE of the pulmonary vascular endothelial surface inactivates bradykinin entirely through hydrolytic degradation during lung passage. Bradykinin is closely associated with anaphylactoid reactions to contrast agents [33].

\section{Influence on blood cells}

The influence of XCM on red and white blood cells, on thrombocytes and the blood coagulation system is complex and not yet fully understood. In vivo and in vitro studies often show divergent results. 
Red blood cells

XCM cause variable, concentration-dependent osmotic shrinkage of erythrocytes, which is related to physicochemical features of the substance and also to its chemical toxicity. Due to the osmotic effect of the hypertonic blood-CM mixture, potassium and liquid pass from erythrocytes into the plasma. Metrizamide or iodipamide solutions of low osmolality modify the membrane of erythrocytes in such a way that they assume a spiky shape (echinocytes) [34, 35].

This transformation reduces the blood cell's elasticity and thus affects blood flow velocity through small capillaries. This effect is rarely observed after injection of nonionic XCM.

White blood cells

After injection of iopromide and the dimeric ionic ioxaglate, the number of leukocytes dropped in patients who underwent arteriography. Nevertheless, the concentration of elastase, a sensitive marker of neutrophilic activation, remained constant. The reduction of leukocytes is most probably attributable to hemodilution [36].

\section{Thrombocytes}

Chronos et al. [37] were the first to demonstrate in vitro that nonionic XCM are able to cause thrombocyte degranulation. Furthermore, an increased serotonin plasma concentration following a decrease of circulating thrombocytes in blood was observed in vivo after injection of ionic ioxaglate and nonionic iopromide [38]. 
Blood coagulation

Nonionic as well as ionic X-ray contrast agents engage in the blood coagulation system at various levels, they particularly inhibit fibrin polymerization and thrombocyte aggregation. However, nonionic XCM, especially at low concentrations, have markedly weaker effects than ionic XCM of high or low osmolality. The occasional observation of thrombus formation in catheters or syringes during angiographic interventions performed with nonionic XCM led to the misconception that these agents are coagulative. Nonionic contrast media are weaker anticoagulants than ionic CM but still anticoagulants. They interact less with biological processes including coagulation than ionic XCM and are, therefore, better tolerable.

Nonionic contrast media rarely and weakly react with biomacromolecules, thus the effect on prolonging blood coagulation is less marked compared with ionic XCM, as shown by blood examinations in animal models and patients [39, 40, 41]. Independently of the choice of the contrast agent the effect of catheters and syringes as well as the technical performance of the examination in terms of triggering blood coagulation through contact activation has to be observed. 


\section{Histamine release}

The release of histamine from mastocytes in humans can trigger pseudoallergic reactions ranging from harmless skin reactions to life-threatening anaphylactoid shock.

Different nonionic XCM showed lower histamine release from rat mastocytes compared to ionic diatrizoic acid. At a contrast medium concentration of $100 \mathrm{mg} / \mathrm{ml}$, histamine release induced by diatrizoic acid was $77 \%$ as opposed to only $3 \%$ for iopromide (fig. 18). In humans, the effect of XCM on histamine-packed mastocytes of the lung is lower upon rapid intra-arterial injection than upon intravenous administration.

This difference might be attributable to the fact that, after intra-arterial injection, the CM bolus passes other organs and becomes diluted before reaching the lung, whereas an i.v. bolus initially passes the lung capillaries at a high concentration.

Every contrast agent is able to increase the blood plasma's histamine level soon after injection, but only for a few minutes. This increase is not mediated by complement activation [42].

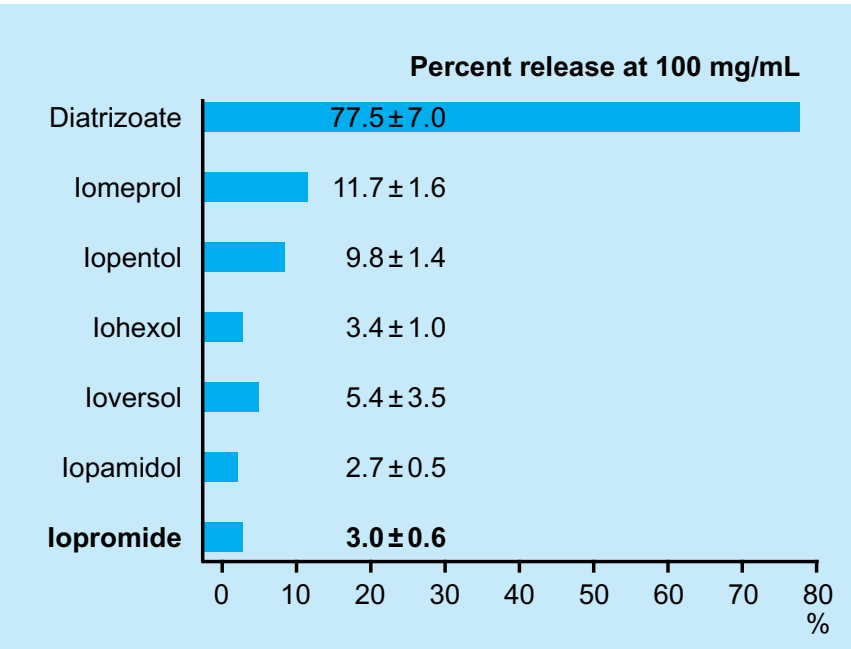

Fig. 18. Histamine release induced by contrast media 


\section{Pharmacological-Toxicological \\ Properties}

For risk assessment of new iodinated X-ray contrast agents, animal experiments had to be performed before clinical trials could be initiated. The toxicological examinations correspond to those used for pharmaceuticals for single administration, i.e.

- Acute toxicity

- Systemic tolerance when applied repeatedly for approximately 4 weeks

- Genotoxicity

- Reproduction toxicity

- Local tolerance

- Examination to detect anaphylactic reactions

lodinated nonionic XCM are usually characterized by a low acute toxicity. The LD50 for dogs, rats and mice after i.v. administration of nonionic XCM is $>10 \mathrm{~g}$ iodine $/ \mathrm{kg}$. Investigations of systemic tolerance after repeated XCM administration are of great importance for risk assessment in humans. Specific organotoxic effects were not found in rats and dogs even after repeat i.v. injection of the highest examined dose $(\geq 2.4-\leq 4 \mathrm{~g}$ iodine $/ \mathrm{kg}$ ) of nonionic XCM such as iotrolan, iohexol, iopromide and iopamidol over a period of 3-4 weeks. Repeated administration (3-5 weeks) of these XCM led to a dose-dependent vacuolization of epithelial cells of the proximal renal tubules starting at a dose of 1.45.0 times the diagnostic dose. Those alterations, which were also observed for ionic contrast agents, did not impair renal function. Electron-microscopic studies with ioxaglate $(7.5 \mathrm{~g}$ iodine $/ \mathrm{kg})$ indicate that high-grade vascuolization of epithelial cells of the proximal renal tubule does not lead to structural alterations of cell organelles [43]. 


\section{Tolerance limits}

Contrast agents are highly concentrated aqueous solutions. A common product contains $150-400 \mathrm{mg} \mathrm{I} / \mathrm{ml}$, corresponding to $300-800 \mathrm{mg}$ iodinated organic molecule. Based on animal experiments of acute toxicity including LD50 (mice, rats), subarachnoid administration, and vascular pain as well as extensive experiences in clinical practice, the following average and maximum doses are proposed for adults. The recommended dose of well-tolerated nonionic XCM in adults is on the order of 1-2 $\mathrm{ml} / \mathrm{kg}$ body weight (BW) (maximum dose $3 \mathrm{ml} / \mathrm{kg} \mathrm{BW})$.

Apart from type and dose of XCM, acute toxicity is also influenced by the injection rate and pre-existing organic diseases, e.g., diabetes mellitus and renal insufficiency. This is to be taken into account when choosing the right dose.

\section{Effects on the cardiovascular system}

Effects on the cardiovascular system can be caused by:

- Osmolality of XCM with rapid ionic and liquid shifts

- Chemical impact on the myocardium and cardiac conduction system

- Release of vasoactive substances.

Rapid intravenous contrast agent injection has a direct impact on vascular walls, leading to vasodilatation and a drop in blood pressure with reflective tachycardia. In coronary angiocraphic examinations, high doses of the contrast agent selectively reach the myocardium, which leads to depressive dose-dependent effects on cardiac contractility. 
High-osmolar CM can cause bradycardia, which lasts longer in ischemic hearts. Causes of cardiotoxic effects were investigated in experiments on dogs.

At 10 -minute intervals, $0.3-0.4 \mathrm{ml} \mathrm{XCM}$ were directly injected into the aortic root of anesthetized laboratory animals until a lethal dose was reached or the experiment was aborted after 15 injections.

Solutions of ionic contrast media interfere with the ionic balance of the myocardium due to high osmolality and binding of calcium [44]. Sodium salts had the highest cardiotoxicity. In a representative experiment, it triggered ventricular fibrillation after only 1-3 injections. Nonionic XCM have lower osmolality and do not bind calcium. Therefore, they induced weaker cardiotoxic reactions such as lower influence on blood pressure in the aorta in animal experiments and are generally better tolerated in clinical coronary angiography [45].

\section{Influence on renal function}

Important mechanisms of nephrotoxicity are disturbances of renal perfusion and damage of glomeruli and tubular cells, which leads to proteinuria. Contrast agents cause these effects to a variable extent. Five tubular enzymes ( $\gamma-\mathrm{GT}, \mathrm{ALP}, \mathrm{LDH}$, AAP, N-acetyl-beta-D-glucosaminidase) showed a significant increase in activity in urine on the first day after injection of high- and low-osmolar contrast media, which indicates damage of tubular cells [46]. A significant reduction of renal blood flow was induced by $10 \mathrm{ml}$ XCM solution in an isolated perfused rat kidney. 
Studies on rats also showed excessive temporary decrease in renal plasma flow after injection of low-osmolar XCM with a concomitant decrease in partial oxygen pressure. No relationship with the osmolality of the contrast agent was identified [47].

Even high doses and concentrations of iodinated contrast media induce acute nephropathy in patients with normal renal function in less than $1 \%$ of cases after i.v. injection and in 2-7\% after intra-arterial injection [48, 49]. However, XCM injection can cause further severe deterioration of renal function in patients with pre-existing renal insufficiency, especially in association with insulin-dependent diabetes, high-grade proteinuria, cardiac insufficiency and high blood pressure. The chemotoxicity of the agent as well as high osmolality and high viscosity of the solution contribute to the development of contrast medium-induced nephropathy. A (usually transient) increase in serum creatinine of more than $25 \%$ or $44 \mu \mathrm{mol} / \mathrm{l}(0.5-1 \mathrm{mg} / \mathrm{dl})$ or a decrease in creatinine clearance of at least $25 \%$ during the first three days after intravascular XCM administration is considered to indicate nephrotoxicity [50].

\section{Allergic and pseudoallergic hypersensitivity reactions}

X-ray contrast media are chemically inert substances, of which high doses (up to $>100 \mathrm{~g}$ ) are administered into the vascular system during a short period of time. The development of nonionic XCM has significantly improved the tolerance of these substances. Nevertheless, hypersensitivity reactions can occur [51]. 
Such reactions are rare and tend to be mild as the most common hypersensitivity reactions are nausea and vomiting. However, bronchospasm, life-threatening drop of blood pressure, cardiac arrest and unconsciousness may occur but are extremely rare. These reactions usually arise during the first 5-10 minutes after injection.

All hypersensitivity reactions are unrelated to the dose or osmolality of the contrast agent given. Early and late hypersensitivity reactions are distinguished (up to $1 \mathrm{~h}$ versus $1 \mathrm{~h}$ to 7 days after XCM injection).

The patient's symptoms do not allow differentiation of allergic from pseudoallergic reactions. Because nonallergic and allergic symptoms are rather similar, terms like anaphylactoid, pseudoallergic or allergy-like are used to describe these reactions.

During the occurrence of immediate hypersensitivity reactions, be it IgE-mediated allergic or nonallergic reactions, the release of histamine has a central role besides other mediators such as prostaglandins, leukotrienes and cytokines. It is known today that an IgE-mediated allergy to XCM is extremely rare $[52,53]$.

The molecular size and weak protein binding of nonionic contrast media almost completely prevent the formation of antibodies in vivo and even in animals under experimental conditions.

Other processes involved in the development of pseudoallergic reactions include activation of the complement system and the contact system (plasma contact system). 
The release of histamine can be mediated by direct contact of contrast agents with mastocytes and basophilic granulocytes or by indirect complement activation with formation of cleavage products $\mathrm{C} 3 \mathrm{a}$ and C5a. The active peptides C3a and C5a increase the permeability of capillaries and venules, which explains the reduction of intravascular volume during severe hypersensitivity reactions.

Administration of contrast media can lead to endothelial damage, which activates the contact system. The contact system includes the blood coagulation system, the fibrinolytic system and the prekallikrein-kallikrein system. Activation of factor $\mathrm{XI}$ stimulates the transformation of prekallikrein to kallikrein. Kallikrein in turn is able to cleave kinins in blood plasma, resulting in formation of the vasoactive compounds bradykinin and lysyl-bradykinin Bradykinin, for instance, inherits histamine-like characteristics.

All of these reactions are quite complex and are controlled by many factors and inhibitors. For example, the shortage of C1-esterase inhibitor seems to play a role in activation of the complement system and contact system. The enzyme C1-esterase, which activates $\mathrm{C} 1$, i.e., the first component of the complement system, can be inhibited by C1-esterase inhibitor.

Late hypersensitivity reactions were not encountered until the advent of nonionic dimeric contrast agents about 30 years ago [54]. These reactions are mostly mild to moderate skin reactions with and without pruritus and, less commonly, headaches, pain at the injection site, gastrointestinal conditions and flu-like symptoms. 
They are most often observed between six hours and three days after i.v. administration of XCM. While such late reactions may be quite unpleasant and difficult to treat, they mostly vanish after three to seven days [55].

Different pathological mechanisms have been proposed to explain the development of late skin reactions. It is believed that most of them are T-cell-mediated reactions, an immunological type IV mechanism $[56,57]$.

\section{Endothelial damage}

Endothelial damage can occur at the injection site and other areas exposed to temporarily high contrast medium concentrations such as the blood-brain barrier in cerebral angiography or vessel segments close to stenoses in coronary or peripheral angiography. This kind of damage is primarily caused by the hyperosmolality of the contrast solution but also by its chemotoxicity. In vitro studies have shown reduced proliferation and increased apoptosis of endothelial cells after incubation of human vascular endothelial cells in intravascular XCM solutions. The effect was lowest for nonionic low-osmolar contrast media [58].

Furthermore, XCM can have temporary effects on microcirculation.

After injection of $20 \mathrm{ml}$ iopromide via a subclavian artery catheter, the flow velocity of erythrocytes in finger fold capillaries decreased by $50 \%$ for 2 minutes [59]. 


\section{Nervous system}

Temporary neurotoxic effects such as convulsive conditions can occur in cerebral and spinal angiography when the blood-brain barrier is disrupted by the contrast agent or as a result of pathological conditions allowing the contrast agent to interact directly with nerve tissue. Those rare neurological effects are related to the chemical structure of the contrast agent rather than physicochemical characteristics such as osmolality or viscosity of the contrast medium solution.

A considerable proportion of neurological problems is attributable to the technical execution of the catheter examination and not so much to the use of ionic or nonionic XCM [60, 61].

Neurotoxicity in animals was investigated by assessing damage to the blood-brain barrier after contrast medium administration for cerebral angiography. After direct injection of the contrast agent into a cerebral ventricle alterations in EEG, behavior and other neural activities are observed.

In studies on rabbits, slight blood-brain barrier damage was demonstrated for the nonionic, monomeric agents iohexol and ioversol and for the nonionic, dimeric agents iodixanol and iotrolan using technetium-99m-pertechnetate as a tracer. A hyperosmolar mannitol solution of $714 \mathrm{mosm} / \mathrm{kg}$ showed less damage, indicating the additional role of chemotoxicity [62].

EEG alterations observed in sedated rabbits after intracerebral injection of iopromide and iohexol differed only lightly between the two agents and did not show any statistically significant difference compared with the effects of an isosmotic mannitol solution [63]. 


\section{Pharmacokinetics}

Two groups of XCM are distinguished on the basis of their pharmacokinetic behavior in the human body: contrast agents that are excreted by the kidneys (CM for computed tomography (CT), angiography and urography) and distribute in the intravascular and extracellular fluid space and hepatocellular or tissue-specific contrast agents (CM for choleangiography).

The water-soluble, iodinated contrast agents used for CT and angiography are not able to pass cell membranes and are mainly eliminated renally. They are not enterally resorbed. After intravascular injection, contrast agents distribute only passively with the blood stream. Their binding to plasma proteins is quite minor ( $<5 \%)$. They enter the interstitial space of most tissues through pores in the capillary wall. Distribution in the intravascular space lasts about 2-3 minutes, whereas the diffusion in the interstitium takes about 10-30 minutes [64]. Contrast agents are eliminated unchanged from the blood through glomerular filtration with an elimination half-life of about 1.5-2 hours.

A much longer elimination half-life of up to 10 hours may be observed in patients with renal insufficiency, which reduces renal clearance to $20 \mathrm{ml} / \mathrm{min}$ and less. Nevertheless, complete elimination was shown for the nonionic iopamidol [65].

Hemodialysis is an effective and safe means to accelerate contrast medium excretion from the body in patients with severe renal insufficiency.

Contrast agents including the cholegraphic media are not able to pass an intact blood-brain barrier. Passage of the placenta and transition into breast milk is markedly restricted. 
A very small proportion of iodine is released from water-soluble, iodinated contrast agents in the human body, even though iodine is strongly bound to the benzene ring. The amount of iodide released from contrast media may reach $3-10 \mathrm{mg}$ per use. There is virtually no risk of interference with thyroid function in healthy patients; however, serious problems may occur in patients with hyperthyroidism. Nevertheless, in all patients, the radioactive iodine uptake test may be altered for weeks after administration of XCM.

The common i.v. choleangiographic agents are composed of two chemically covalently connected tri-iodinated benzoic acid derivates ("dimers"). The molecules are therefore large enough for biliary elimination. The minimum molecular weight for biliary elimination is about 400 Dalton (calculated without the heavy, but small iodine atoms). Intravenous cholegraphics surpass this size without forming conjugates with glucuronic acid whereas the increase in molecular weight through glucuronidation is required for so many other biliarily eliminated substances in the human body and also for the smaller oral cholecystographic agents.

During adequately slow i.v. infusion (10-20 min), iotroxic acid (Biliscopin) is bound to plasma proteins to $80-90 \%$ and is thereby protected from glomerular filtration. A dose of $5 \mathrm{~g}$ iodine is sufficient for ensuring biliary clearance for a minimum of one hour at a maximum transport rate of $0.4 \mathrm{mg} / \mathrm{kg} B W / \mathrm{min}$ in patients with intact liver function. In these patients, $90 \%$ of the contrast agent dose is excreted via feces and only $10 \%$ through the kidneys. XCM reaching the intestine with the bile is not resorbed. 
Open Access This chapter is licensed under the terms of the Creative Commons Attribution 4.0 International License (http://creativecommons.org/licenses/by/4.0/), which permits use, sharing, adaptation, distribution and reproduction in any medium or format, as long as you give appropriate credit to the original author(s) and the source, provide a link to the Creative Commons license and indicate if changes were made.

The images or other third party material in this chapter are included in the chapter's Creative Commons license, unless indicated otherwise in a credit line to the material. If material is not included in the chapter's Creative Commons license and your intended use is not permitted by statutory regulation or exceeds the permitted use, you will need to obtain permission directly from the copyright holder. 